\title{
Use of contrast agents with fast field-cycling magnetic resonance imaging
}

Dara Ó Hógáin ${ }^{1}$, Gareth R Davies ${ }^{1}$, Simona Baroni ${ }^{2}$, Silvio Aime ${ }^{2,3}$ and David J Lurie ${ }^{1}$

${ }^{1}$ Aberdeen Biomedical Imaging Centre, University of Aberdeen, Foresterhill, Aberdeen AB25 2ZD, UK

${ }^{2}$ Invento S.r.1., Via Nizza 52, I-10126, Torino, Italy

${ }^{3}$ Department of Chemistry 'IFM', University of Torino, Italy

E-mail: d.lurie@abdn.ac.uk

\begin{abstract}
Fast Field-Cycling (FFC) MRI allows switching of the magnetic field during an imaging scan. FFC-MRI takes advantage of the $T_{1}$ dispersion properties of contrast agents to improve contrast, thus enabling more sensitive detection of the agent. A new contrast agent designed specifically for use with FFC was imaged using both a homebuilt FFCMRI system and a $3 \mathrm{~T}$ Philips clinical MRI scanner. $T_{1}$ dispersion curves were obtained using a commercial relaxometer which showed large changes in relaxation rate between fields. A model of magnetization behaviour was used to predict optimum evolution times for maximum $T_{1}$ contrast between samples at each field. Images were processed and analysed to create maps of $R_{1}$ values using a set of images at each field. The $R_{1}$ maps produced at two different fields were then subtracted from each other in order to create a map of $\Delta R_{1}$ in which pixel values depend on the change in $R_{1}$ of the sample between the two fields. The dispersion properties of the agent resulted in higher contrast in a $\Delta R_{1}$ image compared with a standard $T_{1}$-weighted image.
\end{abstract}

PACS 87.61.Qr

Physics in Medicine and Biology

Key Words: Fast Field-Cycling, Contrast Agents, Manganese liposomes, $\Delta R_{1}$ imaging 
Dara Ó Hógáin et al - Use of contrast agents with fast field-cycling magnetic resonance imaging

Physics in Medicine and Biology 56, 105-115 (2011)

\section{Introduction}

In contemporary MRI, high magnetic fields (usually above $1.5 \mathrm{~T}$ ) are used to generate high signal-tonoise ratio (SNR) (Redpath 1998). However, the $T_{1}$ values of different tissue types often tend to converge at higher field strengths (above $1.5 \mathrm{~T}$ ), resulting in inherently lower $T_{1}$ contrast (Rinck et al 1988). While contrast agents can be used to improve the contrast between normal and pathological tissue, the relaxivity of the contrast agent is also dependent on magnetic field strength and tends to be lower at higher field strengths (Rinck et al 1988, Carlson et al 1992, Young et al 1981, Bloembergen 1957, Lauffer 1987). Clinical MRI scanners maintain a constant magnetic field $B_{0}$, so that image contrast is inevitably restricted to that which results from differences in tissue NMR parameters (especially $T_{1}$ ) at the scanner's fixed field. On the other hand, Fast Field-Cycling MRI (FFC-MRI) allows the field to be switched between two or more values in a time less than the $T_{1}$ of the sample, always returning to the same magnetic field for signal detection (Carlson et al 1992, Lurie et al 1998, Lurie et al 2010). Thus images can be produced at the magnetic field strength that optimizes contrast enhancement from contrast agents (Rinck et al 1988, Carlson et al 1992). This is done by analysing the dispersion curves of contrast agents and determining the field at which relaxivity $\left(r_{1}\right)$ is highest. Furthermore, FFC-MRI allows access to a new type of contrast known as $\Delta R_{1}$ contrast, in which signal intensity is based on the change in $R_{1}$ of a sample between different magnetic fields (Alford et al 2009).

This work examines the potential of a new contrast agent, designed for molecular imaging applications using FFC-MRI. Molecular imaging investigations usually require probes that can be detected at concentrations in the nano- or even pico-molar range. The agent used here takes the form of a liposome encapsulating $\mathrm{Mn}[\mathrm{II}]$ ions in its inner aqueous cavity. Liposomes containing a large amount of paramagnetic $\mathrm{Mn}[\mathrm{II}]$ ions are expected to display the high relaxation enhancement necessary to tackle the sensitivity issues present in MR molecular imaging assays (Aime et al 2009). The advantage of this contrast agent is the large change in its $r_{1}$ value from a high value at low fields (below $5 \mathrm{mT}$ ) to low $r_{1}$ at higher fields (above $60 \mathrm{mT}$ ), resulting in a large signal value in a $\Delta R_{1}$ image. A comparison is made between $\Delta R_{1}$ mapping using FFC-MRI and other imaging methods, including $T_{1}$-weighted imaging and $T_{1}$ mapping at $3 \mathrm{mT}, 59 \mathrm{mT}$ and at a standard clinical field of $3 \mathrm{~T}$. Contrast is seen to improve using $\Delta R_{1}$ mapping. Moreover it is expected that such a system may show both good specificity and in vivo tolerability, as liposomes are commonly used as drug delivery agents to target diseased tissue and living organisms should be able to control the small excess of essential Mn[II] ions. 
In order to demonstrate proof of purpose of this novel technique, it was considered sufficient to carry out experiments in vitro. The results show how contrast agent conspicuity can be improved using FFC-MRI. Future studies are planned which will show in vivo validation of this new technique at carefully selected magnetic fields.

\section{Magnetization Behaviour during field cycling}

The contrast in a $T_{1}$-weighted inversion-recovery image is largely dependent on the inversion time (TI) used. To optimize the contrast between two samples it is important to understand how the magnetization behaves with inversion time during a pulse. For a fixed-field inversion-recovery pulse sequence the magnetization behaviour can be described by the equation below.

$M(T I)=M_{0}\left(1-2 \exp \left(\frac{-T I}{T_{1}}\right)\right)$

Where $M(T I)$ is the magnetization at a time $(T I)$ after the inversion pulse, $M_{0}$ is the equilibrium magnetization at the readout field, and $T_{1}$ is the longitudinal relaxation time constant of the sample. The optimum inversion time for maximum contrast between two samples with $T_{1}$ values $T_{1 \mathrm{a}}$ and $T_{1 \mathrm{~b}}$ can be determined using the equation below (Ahrens et al 1998).

$T I_{o p t}=\frac{T_{1 a} \cdot T_{1 b}}{\left(T_{1 b}-T_{1 a}\right)} \ln \left(\frac{T_{1 b}}{T_{1 a}}\right)$

However magnetization behaves differently during field-cycling, as the $T_{1}$ value changes between one field and another, thus magnetization evolves at different rates at each field as shown in figure 1 . 


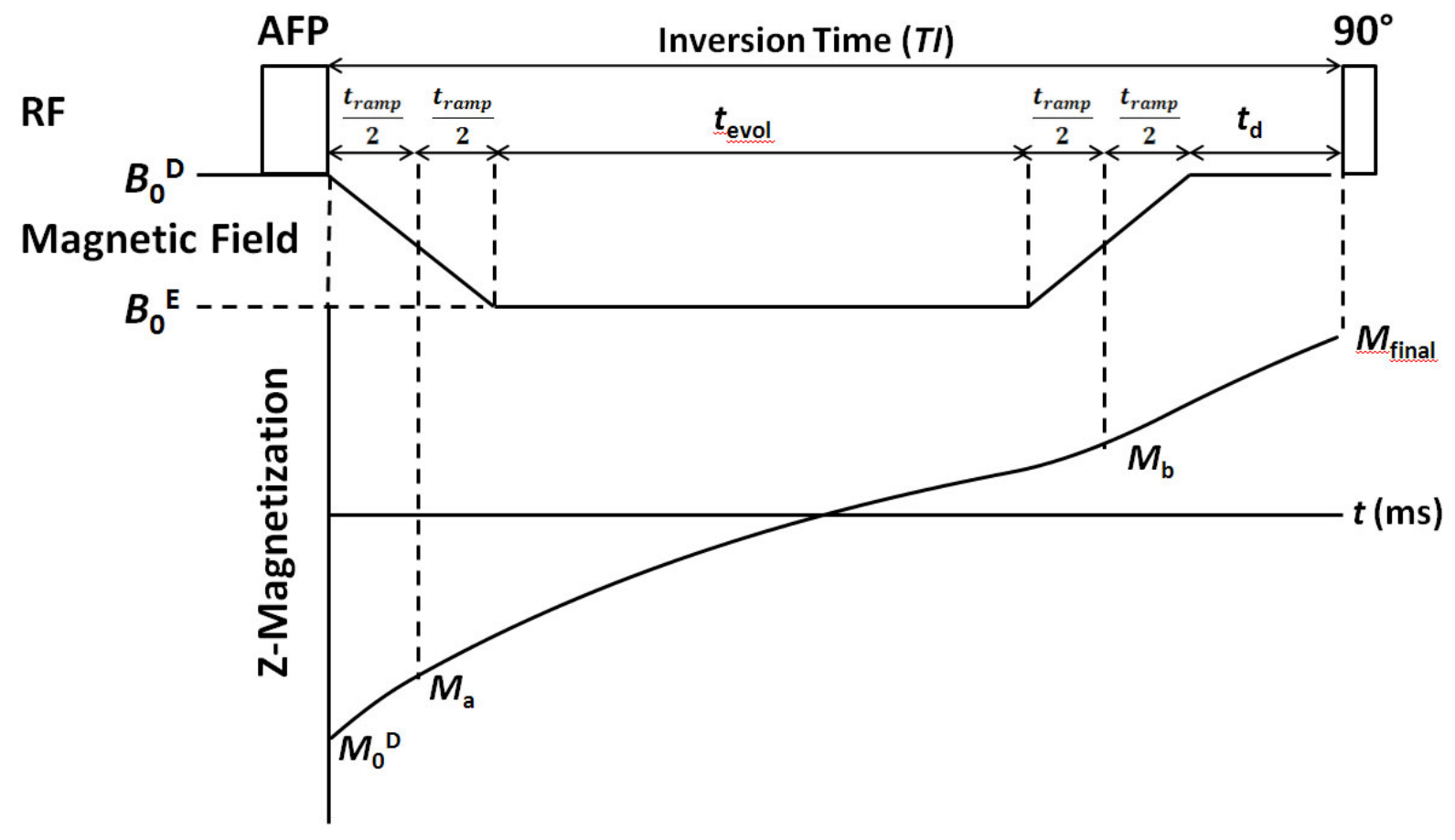

Figure 1: Field-cycling inversion recovery pulse sequence, and magnetization curve, with magnetization values identified at four points $M_{0}^{\mathrm{D}}, M_{\mathrm{a}}, M_{\mathrm{b}}$ and $M_{\text {final }}$.

Following inversion by adiabatic fast passage (AFP) the magnetic field is ramped rapidly (in a time $t_{\text {ramp }} \approx 40 \mathrm{~ms}$ ) from the readout field $\left(B_{0}{ }^{\mathrm{D}}\right)$, to a selected evolution field $\left(B_{0}{ }^{\mathrm{E}}\right)$. The sample's $T_{1}$ value at these fields is designated as $T_{1}{ }^{\mathrm{D}}$ and $T_{1}^{\mathrm{E}}$ respectively.

The magnetization of the sample is allowed to evolve at the evolution field for a time period $\left(t_{\mathrm{evol}}\right)$. The system then ramps back up to the acquisition field followed by a delay time $\left(t_{\mathrm{d}}\right)$ in order for the magnet to stabilize before a $90^{\circ}$ pulse is applied. This is then followed by a gradient echo imaging sequence. The magnetization evolves continuously during the ramp time $t_{\text {ramp }}$, the evolution time $t_{\text {evol }}$ and the delay time $t_{\mathrm{d}}$. As the ramp time is short relative to the evolution time, the ramp between the two fields can be considered as a step process with half of the time spent at the higher field, and half the time at the lower field. The magnetization can be split into four separate stages during the pulse sequence; $\mathrm{M}_{0}{ }^{\mathrm{D}}, M_{\mathrm{a}}, M_{\mathrm{b}}$ and $M_{\text {final }}$ as shown in figure $1 . \mathrm{M}_{\mathrm{a}}$ is given by 


$$
M_{a}=M_{0}^{D}\left(1-2 \exp \left(-\frac{\left(t_{\text {ramp }} / 2\right)}{T_{1}^{D}}\right)\right)
$$

where $\mathrm{M}_{0}{ }^{\mathrm{D}}$ is the equilibrium magnetization at the readout field. $\mathrm{M}_{\mathrm{b}}$ is described by

$$
M_{b}=M_{0}^{E}-\left(M_{0}^{E}-M_{a}\right) \exp \left(-\frac{\left(t_{\text {evol }}+t_{\text {ramp }}\right)}{T_{1}^{E}}\right)
$$

In this case $\mathrm{M}_{0}{ }^{\mathrm{E}}$ is the equilibrium magnetization at the evolution field obtained using the ratio, $B_{0}{ }^{\mathrm{D}} / B_{0}{ }^{\mathrm{E}}=$ $M_{0} / M_{0}^{\mathrm{E}} \cdot M_{\text {final }}$ is the final magnetization described by

$$
M_{\text {final }}=M_{0}^{D}+\left(M_{b}-M_{0}^{D}\right) \exp \left(-\frac{\left(t_{d}+t_{\text {ramp }} / 2\right)}{T_{1}^{D}}\right)
$$

Substituting $M_{\mathrm{a}}$ and $M_{\mathrm{b}}$ into $M_{\text {final }}$ gives a full description of the magnetization behaviour during an FFC inversion recovery pulse sequence.

$M_{\text {final }}=M_{0}^{D}+\left\{M_{0}^{E}-M_{0}^{D}-\left\{M_{0}^{E}-M_{0}^{D}+2 M_{0}^{D} \exp \left[-\frac{\left(t_{\text {ramp }} / 2\right)}{T_{1}^{D}}\right]\right\} \exp \left[-\frac{\left(t_{\text {evol }}+t_{\text {ramp }}\right)}{T_{1}^{E}}\right]\right\} \exp \left[-\frac{\left(t_{d}+t_{\text {ramp }} / 2\right)}{T_{1}^{D}}\right]$

This equation was used to predict magnetization behaviour for a number of different samples with a range of $T_{1}$ time constants at different fields. To validate the equation a comparison was made with experimental data. Using this equation it was possible to select the evolution times which would give maximum contrast between any two samples using $T_{1}$-weighted imaging techniques at different field strengths.

\section{Methods}

\subsection{Sample Preparation}

All the phospholipids used in the liposome preparation were purchased from Avanti Polar; $\mathrm{MnCl}_{2}$ was purchased from Sigma Aldrich. Mn(II)-loaded liposomes were prepared by using a mixture of phospholipids $\left(\mathrm{POPC}=1\right.$-palmitoyl-2-oleoyl-sn-glycero-3-phosphocholine, $95 \%$ and $\mathrm{DSPE}^{-\mathrm{PEG}_{2000}=}$ 
Dara Ó Hógáin et al - Use of contrast agents with fast field-cycling magnetic resonance imaging

Physics in Medicine and Biology 56, 105-115 (2011)

1,2-distearoyl-sn-glycero-3-phospoethanolamine-N-[metoxy(polyethylene glycol)-2000), 5\%). The thin lipidic method was followed (Lasch et al 2003). Briefly, the lipids (about $30 \mathrm{mg} / \mathrm{ml}$ ) were dissolved in chloroform and the organic solution was slowly evaporated for removing the solvent until a thin film was formed. The film was then hydrated at $55{ }^{\circ} \mathrm{C}$ with a $5 \mathrm{mM}$ aqueous solution of $\mathrm{MnCl}_{2}$, whose osmolarity was corrected to $300 \mathrm{mOSm}$ with $\mathrm{NaCl}$. The resulting suspension of multilamellar vesicles (MLV) was extruded (Lipex extruder, Northern Lipids Inc., Canada) through progressively smaller pore sizes (polycarbonate filters with pore diameters of 400, 200 and $100 \mathrm{~nm}$, respectively). The final suspension of liposomes was purified from the not encapsulated metal ions by exhaustive dialysis against a HEPES (N2-hydroxyethylpiperazine-N'-2-ethanesulfonic acid)/ $\mathrm{NaCl}$ buffer ( $\mathrm{pH} 7.4,300 \mathrm{mOsm}$ ). The liposomes were characterized by dynamic light scattering (Zetasizer NanoZS, Malvern, UK) in order to assess the mean hydrodynamic size and the polydispersity of the system. The liposomes used in this work showed an average diameter of $111 \mathrm{~nm}$; the polydispersity index (PDI) was smaller than $0.1 . \mathrm{Mn}[\mathrm{II}]$ concentration in the liposome suspension was determined by $T_{1}$ measurement after the disruption of the liposomes' membranes by mineralization with $\mathrm{HCl}$. A stock liposome suspension corresponding to a total $\mathrm{Mn}[\mathrm{II}]$ concentration of $0.77 \mathrm{mM}$ was used for the preparation of an imaging phantom.

The suspension was diluted in HEPES/ $\mathrm{NaCl}$ buffer solution in order to prepare three samples at the following concentrations: A: $0.8 \mathrm{mM}, \mathrm{B}: 0.045 \mathrm{mM}, \mathrm{C}: 0.035 \mathrm{mM}$. A solution of $0.23 \mathrm{mM}$ Prohance was also prepared in de-ionized water in order to provide comparison with the $\mathrm{Mn}[\mathrm{II}]$ contrast agent. Prohance is a standard contrast agent comonly used in clinical MRI scans but unlike Mn[II] shows very little change in $R_{1}$ between field strengths.

\subsection{Dispersion Curve Measurement}

The samples were placed in NMR tubes and dispersion curves were measured. The dispersion curve shape of each sample depends on a combination of factors including; the concentration of the agent in the solution, the concentration of the entrapped paramagnetic molecule within the liposome, the type of paramagnetic species, the viscosity of the hydration solution, which gives rise to a peak at high fields, the membrane composition which affects the water exchange time, and the total size of the liposome, which relates to the reorientational correlation time.

Dispersion curves were obtained experimentally using a field-cycling pulse sequence on a commercial NMR relaxometer (Stelar S.r.l., Italy). Pre-polarization was typically at $187 \mathrm{mT}$, with detection at 169 
mT. At each value of the evolution field the sequence was repeated using $\mathrm{N}$ different values of the inversion time (TI), allowing $T_{1}$ to be calculated as a function of magnetic field (Ferrante and Sykora 2004).

\subsection{Imaging}

An imaging phantom was constructed containing samples of $\mathrm{Mn}[\mathrm{II}]$ contrast agent solutions arranged alongside a $0.23 \mathrm{mM}$ sample of Prohance. The samples were contained in glass tubes with dimensions 1 $\mathrm{cm}$ diameter x $2 \mathrm{~cm}$ length. Samples were imaged on both a homebuilt whole-body FFC-MRI system (Lurie et al 1998), and a 3 T Philips Achieva clinical MRI scanner. The FFC-MRI system employed two coaxial magnets. The primary permanent magnet provides a homogeneous fixed detection field of 58.7 $\mathrm{mT}$ (2.5 MHz proton frequency), while the secondary resistive electromagnet provided a variable offset field, capable of ramping from 0 to $58.7 \mathrm{mT}$ in $40 \mathrm{~ms}$ (Lurie et al 1998). During the ramp time the magnetic field changes linearly with time until the desired evolution field strength is reached. Following the ramp time there is a brief period of slight magnetic field instability $(<1 \mathrm{mT})$ caused by the time it takes the amplifier to switch off completely as well as by eddy currents induced in the coil. This instability occurs over a short time period and has negligible effect on the final bulk magnetization as described by equation 6 . However a short delay has been implemented following field cycling ramps and before any RF pulse are applied, so that this instability does not affect the RF pulse efficiency. The total homogeneity of the evolution field was within $500 \mathrm{ppm}$. The stability of the evolution field is dependant on the current supply to the electromagnet, and has been found to vary by up to $1 \%$ which is stable enough not to affect the $T_{1}$ value of the sample.

3.3.1. $T_{1}$ weighted imaging. $T_{1}$ weighted images were acquired using the FFC-MRI scanner at $59 \mathrm{mT}$ and $3 \mathrm{mT}$ using a range of evolution times at each field, with evolution times carefully selected to show the maximum contrast between each $\mathrm{Mn}[\mathrm{II}]$ sample and the Prohance sample. The inversion time following an inversion pulse is equivalent to the evolution time plus an additional $150 \mathrm{~ms}$ (which includes the time needed for both the ramp times (each $40 \mathrm{~ms}$ duration) plus the delay time (70 ms) before the $90^{\circ}$ pulse. The fields were selected to show maximum change in $R_{1}$ of the $\mathrm{Mn}[\mathrm{II}]$ contrast agent. An FFC inversion recovery imaging pulse sequence was used as shown in figure 1, but with the addition of imaging 
gradients and a selective $90^{\circ}$ pulse. The matrix size was 64 x 64, with number of excitations (NEX) $=1$. Image slice thickness was $0.8 \mathrm{~cm}$, and the field of view (FOV) was $8 \mathrm{~cm}$.

$T_{1}$-weighted images were also acquired at $3 \mathrm{~T}$ using a Philips clinical MRI scanner, with a slice thickness of $0.8 \mathrm{~cm}$ and an FOV of $6 \mathrm{~cm}$. The imaging phantom was placed in a knee coil and images were produced using a standard spin-echo inversion-recovery pulse sequence with a range of inversion times. The samples' $T_{1}$ times were much longer at $3 \mathrm{~T}$ compared with $59 \mathrm{mT}$, thus a much wider range of inversion times was selected to produce an accurate $R_{1}$ map.

3.3.2. $\Delta R_{1}$ mapping. A disadvantage of $T_{1}$-weighted imaging in FFC-MRI is that the signal is dependent not only on the $T_{1}$ value of the sample but also on the equilibrium magnetization at the evolution field $\left(\mathrm{M}_{\mathrm{e}}\right)$ (Ungersma et al 2006, Alford et al 2009). The intensity values shown in a $\Delta R_{1}$ map however depend solely on the change in $R_{1}$ between fields and can take full advantage of the dispersion characteristics of the contrast agent. $\Delta R_{1}$ mapping is a similar technique to $T_{1}$ mapping which is used in MRI to allow quantitative analysis of the distribution of contrast agents in vivo (Treier et al 2008). This technique first processes a series of images taken at multiple evolution times, and creates a map of $R_{1}$ values for each pixel. $R_{1}$ maps created at different fields can then be subtracted from each other to create a map of $\Delta R_{1}$ values, representing the change in $R_{1}$ of the samples between the two fields. 


\section{Results}

\subsection{Dispersion Curve Measurement}

Figure 2 shows the measured dispersion curve of the $0.77 \mathrm{mM} \mathrm{Mn}[\mathrm{II}]$ sample.

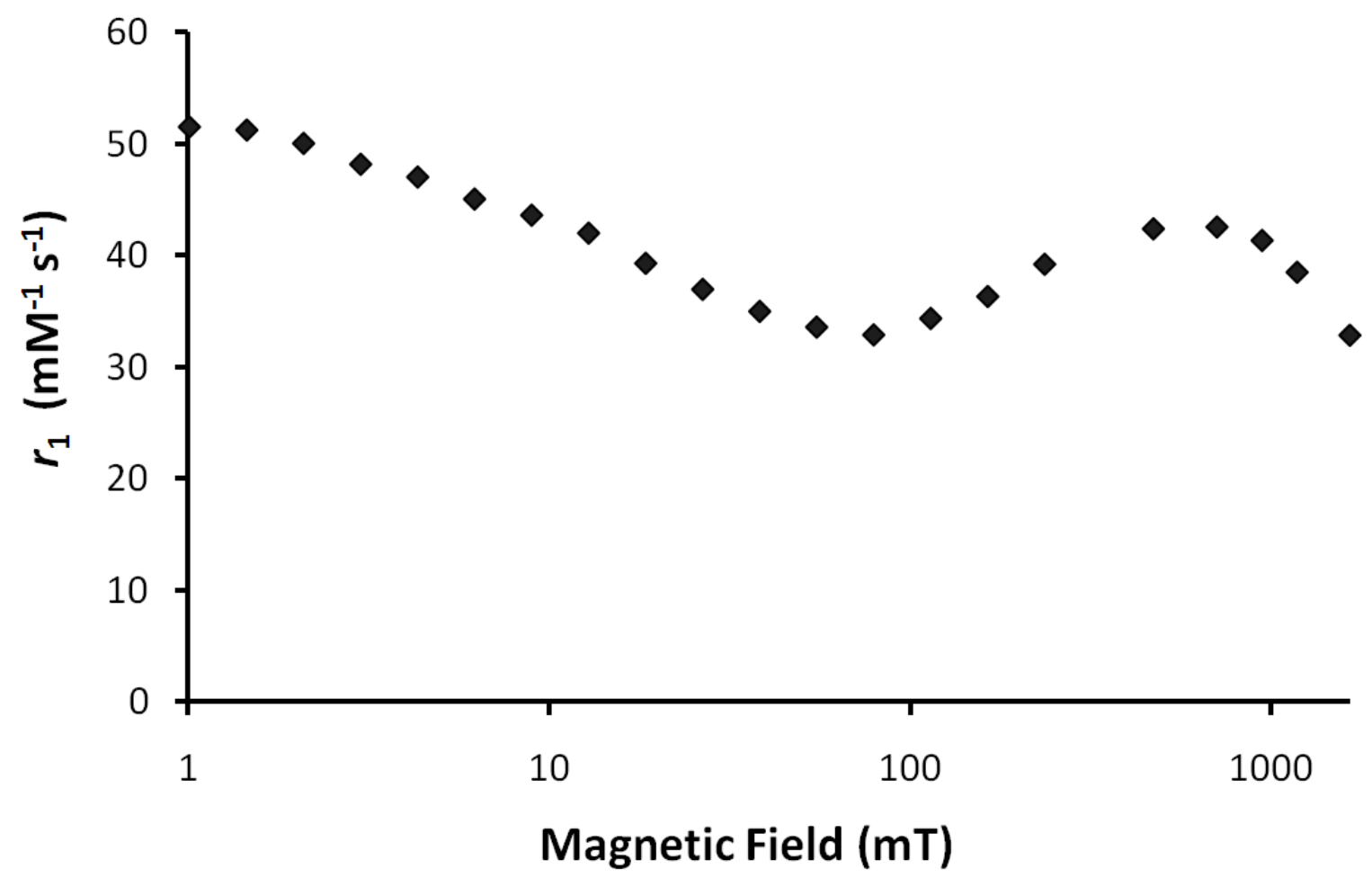

Figure 2: $T_{1}$ dispersion curve of $0.77 \mathrm{mM} \mathrm{Mn[II]} \mathrm{liposome} \mathrm{samples} \mathrm{at} 25^{\circ} \mathrm{C}$. Measured using a Stelar SPINMASTER relaxometer.

The $0.77 \mathrm{mM}$ stock solution was diluted in a HEPES buffer solution and dispersion curves were acquired at different final concentrations of $\mathrm{Mn}[\mathrm{II}]$ as shown in figure 3; a dispersion curve of $0.23 \mathrm{mM}$ Prohance solution is also shown. 


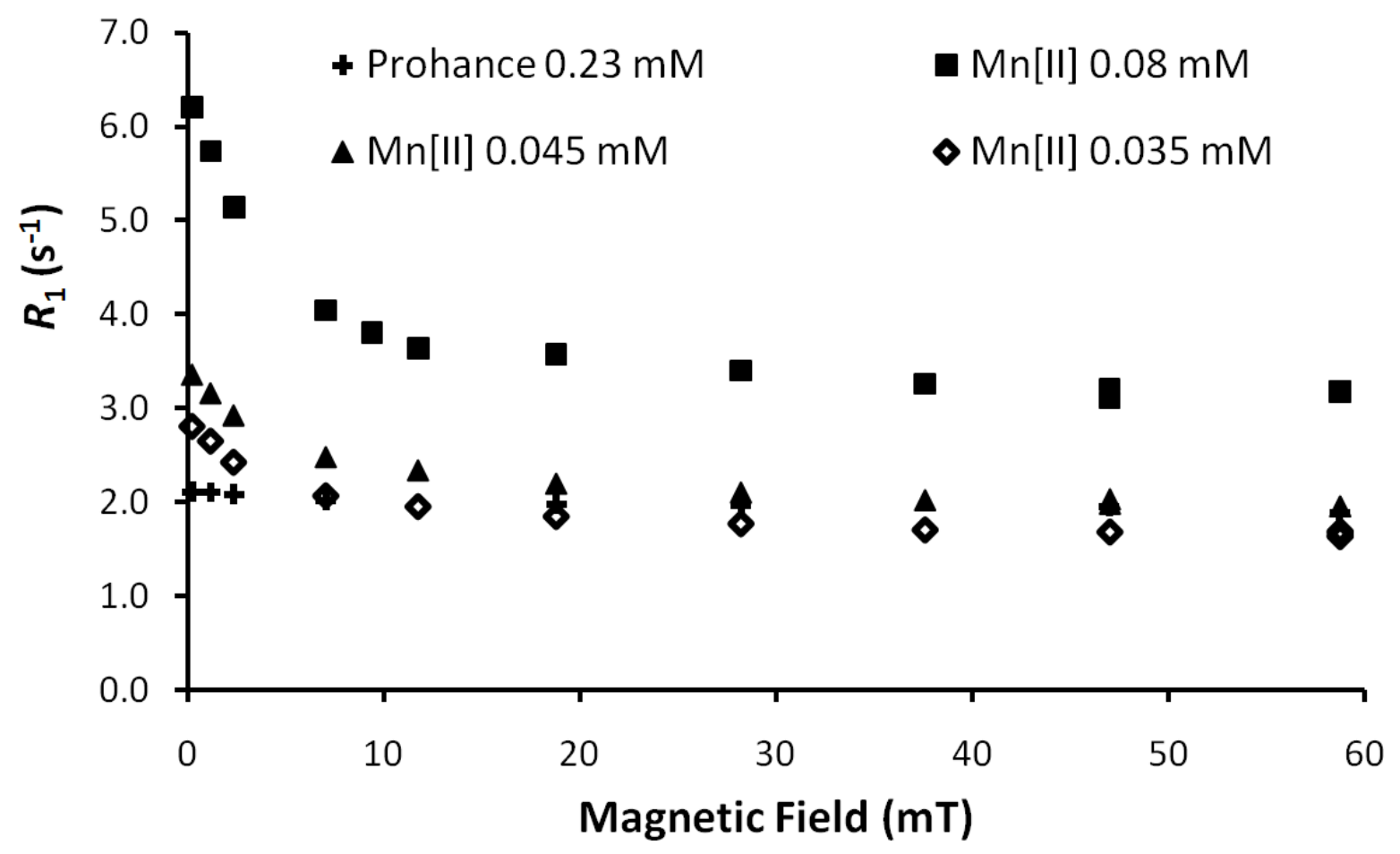

Figure 3: $T_{1}$ dispersion curves of $\mathrm{Mn}[\mathrm{II}]$ Liposomes and Prohance sample at $25^{\circ} \mathrm{C}$. Measured using a Stelar SMARtracer relaxometer.

The sample with the highest concentration of $\mathrm{Mn}[\mathrm{II}]$ shows a change of $\sim 3 \mathrm{~s}^{-1}$ between $3 \mathrm{mT}$ and $59 \mathrm{mT}$, whereas the $0.23 \mathrm{mM}$ Prohance sample only shows a change of $\sim 0.2 \mathrm{~s}^{-1}$ over the same field range. Thus the difference between these samples should be clearly distinguishable in a $\Delta R_{1}$ map.

\section{2. $T_{1}$ weighted imaging}

An imaging phantom containing samples of $\mathrm{Mn}[\mathrm{II}]$ liposomes and a sample of Prohance was imaged at $59 \mathrm{mT}$ with an inversion time of $750 \mathrm{~ms}$ as shown in figure 4 (a). The imaging phantom was then imaged at a range of inversion times at both $59 \mathrm{mT}$ and $3 \mathrm{mT}$ as shown in figure 4 (b) and (c) respectively, and finally at 3 T using a Philips clinical MRI scanner as shown in figure 4 (d). 
(a)

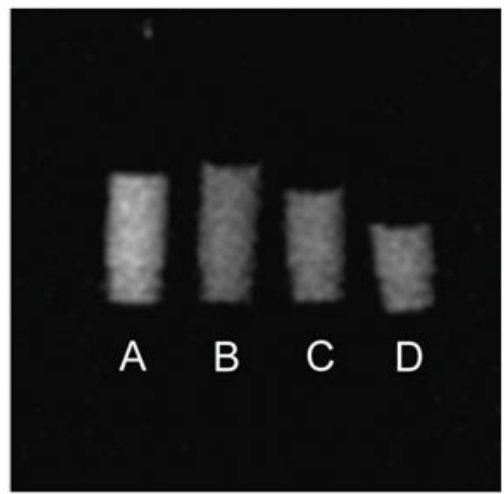

(b)
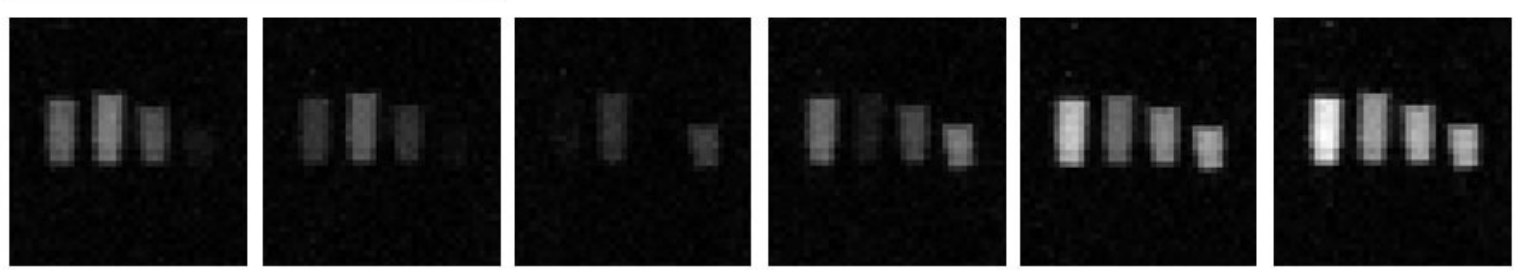

(c)
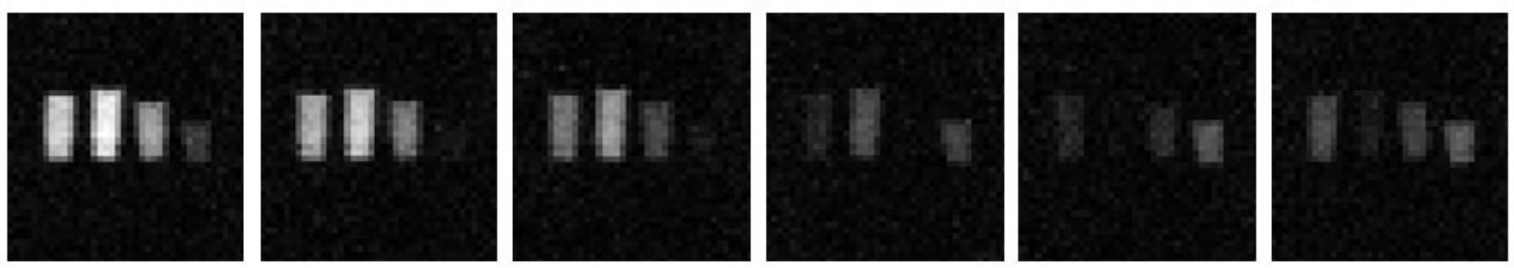

(d)
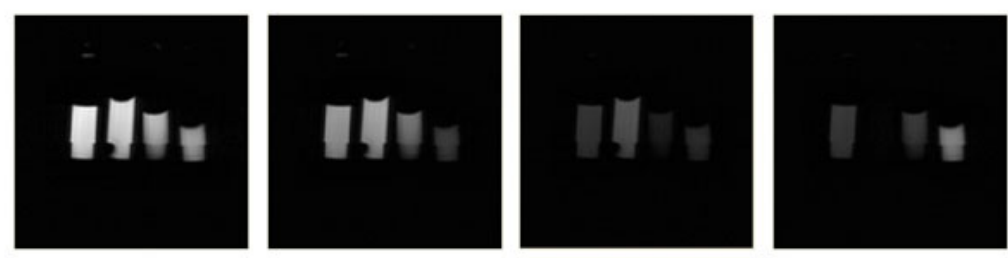

(i)

(ii)

(iii)

(iv)

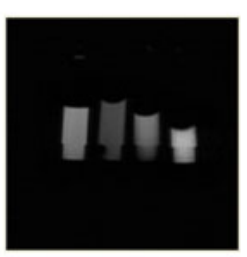

(v)

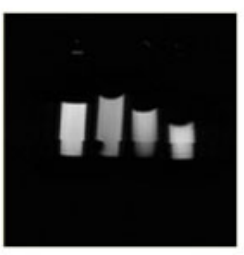

(vi)

Figure 4: Phantom image, obtained using an FFC inversion recovery pulse sequence (a) at $59 \mathrm{mT}$ with inversion time of $750 \mathrm{~ms}$ A: Prohance $0.23 \mathrm{mM}, \mathrm{B}: \mathrm{Mn}[\mathrm{II}] 0.08 \mathrm{mM}, \mathrm{C}: \mathrm{Mn}[\mathrm{II}] 0.05 \mathrm{mM}, \mathrm{D}: \mathrm{Mn}[\mathrm{II}] 0.03$ mM. (b) $59 \mathrm{mT}$ at a range of inversion times: (i) $190 \mathrm{~ms}$ (ii) $240 \mathrm{~ms}$ (iii) $370 \mathrm{~ms}$ (iv) $590 \mathrm{~ms}$ (v) $850 \mathrm{~ms}$ (vi) 1150. (c) $3 \mathrm{mT}$ at a range of evolution times: (i) $190 \mathrm{~ms}$ (ii) $240 \mathrm{~ms}$ (iii) $370 \mathrm{~ms}$ (iv) $590 \mathrm{~ms}$ (v) 850 ms (vi) 1150 ms. Imaging parameters are as follows: matrix size $=64 \times 64, \mathrm{NEX}=1$, Repetition time $=$ $1.5 \mathrm{~s}$, Field of view $=8 \mathrm{~cm}$, slice thickness $=0.8 \mathrm{~cm}$. Total imaging time was 63 minutes. (d) $3 \mathrm{~T}$ at a range of inversion times (i) $50 \mathrm{~ms}$ (ii) $200 \mathrm{~ms}$ (iii) $400 \mathrm{~ms}$ (iv) $700 \mathrm{~ms}$ (v) $950 \mathrm{~ms}$ (vi) 1600. Imaging parameters were as follows: slice thickness $0.8 \mathrm{~cm}$, FOV $6 \mathrm{~cm}$, total imaging time for all images was 20 minutes. 


\subsection{Magnetization Model}

A region of interest was selected for each sample in all of the $T_{1}$ weighted images, and the mean signal and standard deviation were measured. The mean signal was then plotted against evolution time. This experimental data was then compared with predicted magnetization curves (figure 5) obtained using equation 6. For clarity, only curves from two samples are shown corresponding to (a) $\mathrm{Mn}[\mathrm{II}] 0.8 \mathrm{mM}$ with $T_{1}$ of $181 \mathrm{~ms}$ at $3 \mathrm{mT}$ and (b) Prohance $0.23 \mathrm{mM}$ with $T_{1}$ of $471 \mathrm{~ms}$ at $3 \mathrm{mT}$. Values for $T_{1}{ }^{0}$ and $T_{1}^{\mathrm{e}}$ used in equation 6 were obtained from relaxometry data, and $M_{e}$ was calculated using the ratio $B_{0} / B_{e}=M_{0} / M_{e}$.
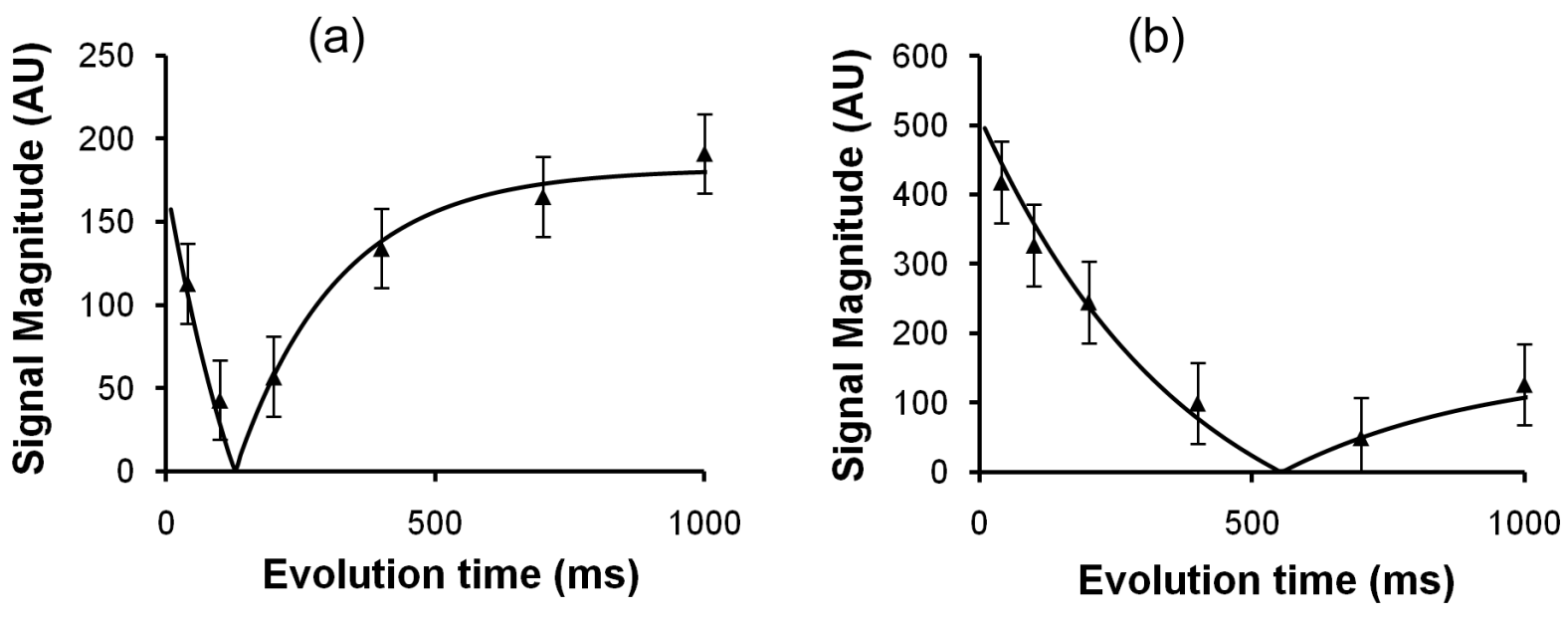

Figure 5: A comparison between experimental (triangle) and predicted (solid line) signal magnititude vs. evolution time at $3 \mathrm{mT}$ for (a) $0.08 \mathrm{mM} \mathrm{Mn[II]} \mathrm{sample,} \mathrm{and} \mathrm{(b)} 0.23 \mathrm{mM}$ Prohance sample.

The experimental data was seen to match the predicted magnetization curve within experimental error, thus demonstrating that equation 6 could be used to predict magnetization behaviour during a fieldcycling inversion-recovery pulse sequence. The model could therefore be used to predict contrast as a function of evolution time in order to optimize $T_{1}$-weighted contrast at any evolution field value. 


\section{4. $\Delta R_{l}$ Mapping}

A $\Delta R_{1}$ map was produced by first creating maps of $R_{1}$ values at $59 \mathrm{mT}$ and $3 \mathrm{mT}$. The $R_{1}$ maps were constructed using a program written in MATLAB (MathWorks, Natick, MA, USA) which overlaid a set of $T_{1}$-weighted images acquired at a range of evolution times using an inversion-recovery pulse sequence. The MATLAB program plotted signal intensity against evolution time to determine the $R_{1}$ value for each pixel in the set of images. $R_{1}$ maps were then subtracted to give a map of $\Delta R_{1}$.

$R_{1}$ maps obtained at $59 \mathrm{mT}$ and $3 \mathrm{mT}$ are shown in figures $6 \mathrm{a}$ and $6 \mathrm{~b}$, and a map of $\Delta R_{1}$ values is shown in figure 6c. In the $R_{1}$ maps the contrast between the $\mathrm{Mn}[\mathrm{II}]$ samples and the Prohance sample increases when the field is switched to $3 \mathrm{mT}$ due to the diverging relaxation rates at low field. The $\Delta R_{1}$ map however shows higher contrast between the Mn[II] samples and the Prohance sample than either of the fixed-field $R_{1}$ maps.

The measurement of the $R_{1}$ value was found to have a standard deviation of $\sim 10 \%$ and $\sim 15 \%$ at $59 \mathrm{mT}$ and $3 \mathrm{mT}$ respectively, while the standard deviation for the $\Delta R_{1}$ map is $\sim 25 \%$. Uncertainty is dependent on a number of factors including the inherent signal-to-noise ratio, the variation of the sample magnetization with field, the number of evolution times used, and the number of signal averages used for each image (Ferrante and Sykora 2004).

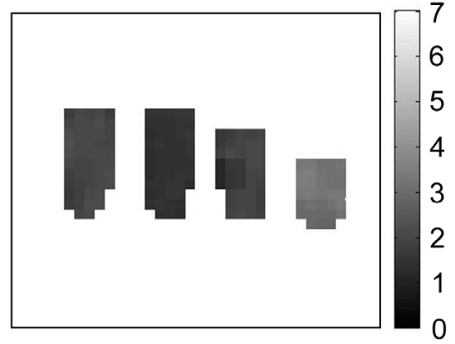

(a)

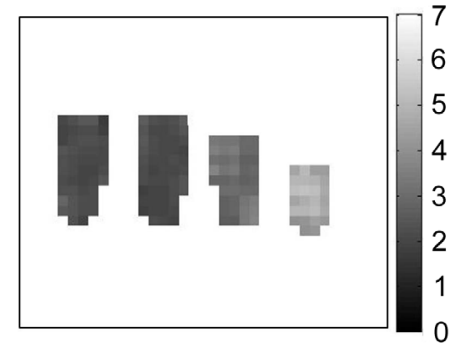

(b)

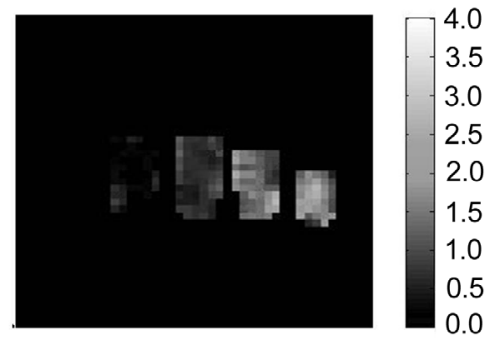

(c)

Figure 6: Sections from $R_{1}$ maps obtained at (a) $59 \mathrm{mT}$, (b) $3 \mathrm{mT}$; (c) $\Delta R_{1}$ map obtained by subtracting $R_{1}$ maps shown in (a) and (b). 


\subsection{Image Analysis}

The contrast between $\mathrm{Mn}[\mathrm{II}]$ liposome and Prohance samples was compared using contrast-optimized $T_{1^{-}}$ weighted imaging at $3 \mathrm{~T}, 59 \mathrm{mT}, 3 \mathrm{mT}$ and $\Delta R_{1}$ mapping. In order to compare contrast using these different imaging modalities the contrast between the $\mathrm{Mn}[\mathrm{II}]$ samples and the Prohance sample was first defined as follows (Hendrick and Raff 1992):

Contrast ratio $(\%)=\frac{\left(S_{A}-S_{B}\right)}{\left(S_{A}+S_{B}\right)} \times 100$

As shown in figure 7, this comparison reveals that contrast is significantly increased using $\Delta R_{1}$ mapping compared with $T_{1}$-weighted imaging. In particular, the contrast ratio between $1.0 \mathrm{mM}$ Prohance and 0.045 $\mathrm{mM} \mathrm{Mn}[\mathrm{II}]$ samples (with $T_{1}$ values of $812 \mathrm{~ms}$ and $765 \mathrm{~ms}$ respectively) in the $T_{1}$-weighted image obtained at $3 \mathrm{~T}$ is $36 \%$. The contrast between these samples increases to $78 \%$ using $\Delta R_{1}$ imaging which shows how the $\mathrm{Mn}[\mathrm{II}]$ agent can be detected with significantly greater sensitivity using $\Delta R_{1}$ mapping. It should be noted that the enhanced contrast is observed using data obtained at $3 \mathrm{mT}$ and $59 \mathrm{mT}$, i.e. between one fiftieth and one thousandth of the field used in the high-field experiment.

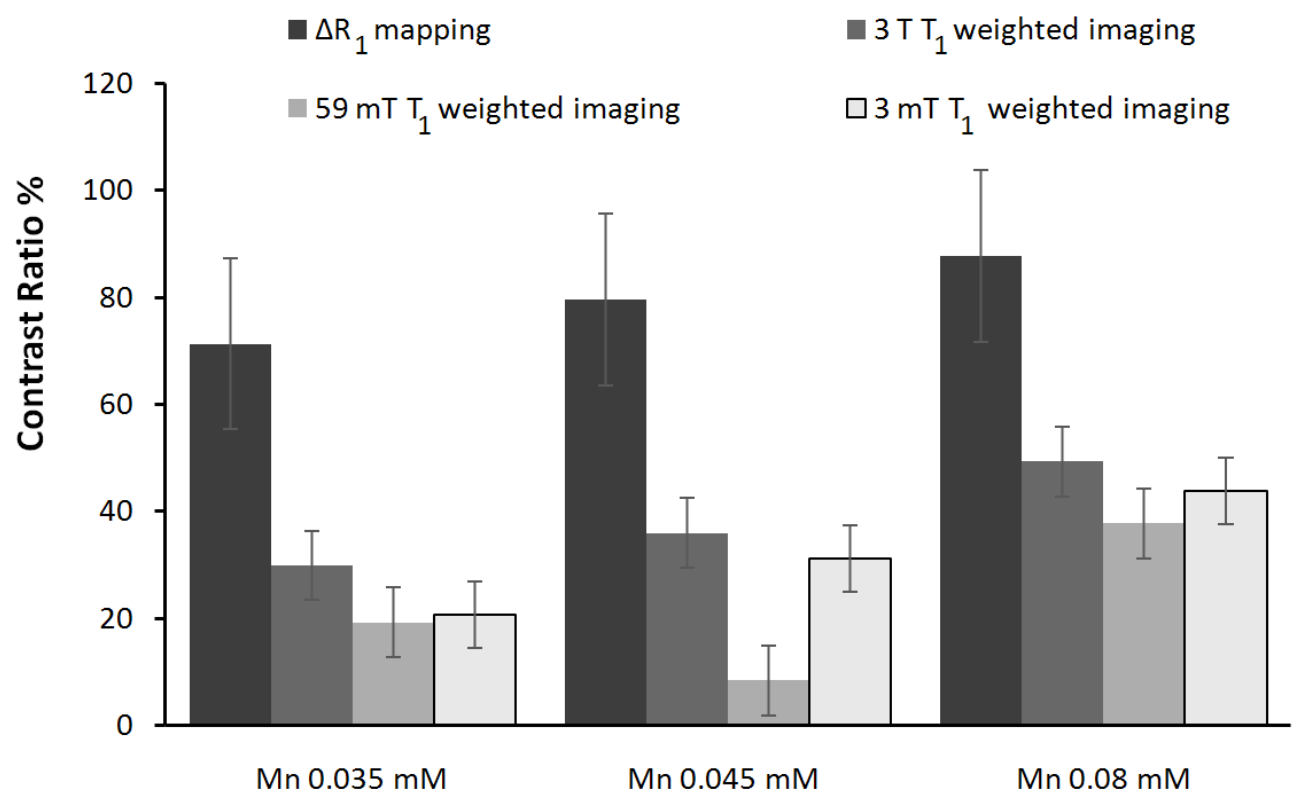

Figure 7: Comparison of contrast ratio from $T_{1}$-weighted images and $\Delta R_{1}$ image. Error bars are based on standard deviation across the images. 


\section{Discussion}

The contrast between different concentrations of a new $\mathrm{Mn}[\mathrm{II}]$ liposome-based contrast agent and a conventional agent (Prohance) was compared using different imaging methods. These imaging methods included $T_{1}$-weighted imaging at $3 \mathrm{mT}, 59 \mathrm{mT}$, and $3 \mathrm{~T}$, and $\Delta R_{1}$ mapping. Multiple images were used to produce a $\Delta R_{1}$ map which allowed a quantitative analysis of the change in $R_{1}$ between different fields. This method suppressed the values from the Prohance sample, increased the sensitivity of detection of the $\mathrm{Mn}[\mathrm{II}]$ agents, and showed that the $\Delta R_{1}$ values were proportional to the concentration of the $\mathrm{Mn}[\mathrm{II}]$ contrast agent. Furthermore the liposome sample used here of $111 \mathrm{~nm}$ diameter contains ca. $10^{3} \mathrm{Mn}$ [II] ions $^{1}$. Thus suspensions containing $0.15 \mathrm{mM}$ and $0.06 \mathrm{mM} \mathrm{Mn}[\mathrm{II}]$ ions correspond to ca. 60 and $30 \mathrm{nM}$ concentration of liposomes, respectively. The observed $\Delta R_{1}$ enhancements clearly indicate that the proposed method (FFC-MRI and dispersion-tailored reporting probe) is well suited for molecular imaging applications. The present FFC-MRI system has shown consistency in its measurements and has provided proof-of-principle that $\Delta R_{1}$ mapping can be used to improve the conspicuity of tailored contrast agents. However the error inherent in $\Delta R_{1}$ mapping remains a significant problem as it reduces the sensitivity of the technique to detect small changes in $R_{1}$. In order to reduce error and improve sensitivity, more averages and more evolution times could be used in image acquisition. Unfortunately this would lead to longer imaging times. At present the time needed to produce multiple images at different field strengths is approximately 63 minutes, which is clearly too long for a clinical scan. However this time may be reduced using a faster $T_{1}$ mapping sequence such as an inversion-recovery Look-Locker echo-planar imaging sequence (Look and Locker 1970, Shin et al 2009).

The main challenge to applying $\Delta R_{1}$ mapping in vivo is to know at which fields to image in order to optimize contrast enhancement in a $\Delta R_{1}$ map. This information can be obtained by acquiring dispersion curves in vivo of tissue with and without the contrast agent. To this end further work has been carried out in parallel to this study, to obtain localized dispersion curves in vivo. (Pine et al, 2009). The relaxation curves of biological tissues have been studied extensively (Bottomley et al 1984, Escanye et al 1982, Fischer et al 1990) and tend to show relatively little change in $R_{1}$ at field strengths above $200 \mathrm{mT}$, whereas the $\mathrm{Mn}$ liposome samples exhibit large changes in $R_{1}$ within this field range (as shown in figure 2). To carry out in vivo experimental work a new FFC-MRI system with a field range between 0 and 0.5

\footnotetext{
${ }^{1}$ The determination of the number of $\mathrm{Mn}[\mathrm{II}]$ per liposome has been assessed by measuring the absorbance of a fluorescent dye added to the hydration solution of Mn[II] ions (Zuidam et al 2003).
} 
Dara Ó Hógáin et al - Use of contrast agents with fast field-cycling magnetic resonance imaging

Physics in Medicine and Biology 56, 105-115 (2011)

tesla has been built in our laboratory and is in its final testing stages. This system will have improved field homogeneity, faster ramp times and a higher detection field, allowing more accurate and improved $\Delta R_{1}$ mapping using FFC.

\section{Acknowledgements}

The authors acknowledge financial support for the FFC-MRI project from Research Councils UK and the Engineering and Physical Sciences Research Council, under the Basic Technology scheme (grant no. EP/E036775/1). They thank Mr. Azlan Bin Che Ahmad for his assistance with the measurements at $3 \mathrm{~T}$. 
Dara Ó Hógáin et al - Use of contrast agents with fast field-cycling magnetic resonance imaging

Physics in Medicine and Biology 56, 105-115 (2011)

\section{References}

Ahrens E T, Rothbächer U, Jacobs R E and Fraser S E 1998 A model for MRI contrast enhancement using $\mathrm{T}_{1}$ agents Proc. Natl. Acad. Sci. USA 95 8443-8

Aime S, Delli Castelli D, Geninatti Crich S, Gianolio E and Terreno E 2009 Pushing the sensitivity envelope of lanthanide based magnetic resonance imaging (MRI) contrast agents for molecular imaging applications Acc. Chem. Res. 42 822-831

Alford J K, Rutt B K, Scholl T J, Handler W B and Chronik B A 2009 Delta relaxation enhanced MR: improving activation-specificity of molecular probes through $\mathrm{R}_{1}$ dispersion imaging Magn. Reson. Med. $61796-802$

Bloembergen N 1957 Proton relaxation times in paramagnetic solutions J. Chem. Phys. 27 572-573

Bottomley P A, Foster T H, Argersinger R E and Pfeifer L M 1984 A review of normal tissue hydrogen NMR relaxation times and relaxation mechanisms from 1-100 MHz Med. Phys. 11425

Carlson J W, Goldhaber D M, Brito A and Kaufman L 1992 MR relaxometry imaging. Work in progress Radiology 184 635-639

Escanye J M, Canet D and Roberta J 1982 Frequency dependence of water proton longitudinal nuclear magnetic relaxation times in mouse tissues at $20^{\circ} \mathrm{C}$ Biochim. Biophys. Acta. 721, 305-311

Ferrante G and Sykora S 2004 Technical aspects of fast field-cycling relaxometry Adv. Inorg. Chem. 57 $405-470$

Fischer H W, Rinck P A, Van Haverbeke Y and Muller R N 1990 Nuclear relaxation of human brain gray and white matter: analysis of field dependence and implications for MRI Magn. Reson. Med. 16, 317-34.

Hendrick R E and Raff U 1992 Magnetic Resonance Imaging (2nd ed.). St. Louis: Mosby. 109-144.

Lasch J, Weissig V and Brandl M 2003 Preparation of liposomes, in Liposomes - A Practical Approach, $2^{\text {nd }}$ edition, ed V P Torchilin and V Weissig (Oxford: Oxford University Press) p 3

Lauffer R B 1987 Paramagnetic metal complexes as water proton relaxation agents for NMR imaging: theory and design Chem. Rev. 87 901-927 
Look D C and Locker D R 1970 Time saving in measurement of NMR and EPR relaxation times. Rev. Sci. Instrum. 41 250-251

Lurie D J, Foster M A, Yeung D and Hutchison J M S 1998 Design, construction and use of a largesample field-cycled PEDRI imager Phys. Med. Biol. 43 1877-1886

Lurie D J, Aime S, Baroni S, Booth N A, Broche L M, Choi C-H, Davies G R, Ismail S, Ó hÓgáin D and Pine K J 2010 Fast field-cycling magnetic resonance imaging Comptes Rendus Physique 11 136-148

Pine K J, Davies G R and Lurie D J 2009. Field-cycling NMR relaxometry with spatial selection. Magn. Reson. Med., 63 1698-1702

Redpath T W 1998 Signal-to-noise ratio in MRI Br. J. Radiol. 71 704-707

Rinck P A, Fischer H W, Vander Elst L, Van Haverbeke Y and Muller R N 1988 Field-cycling relaxometry: Medical applications Radiology 168 843-849

Shin W, Gu H and Yang Y 2009 Fast high-resolution $\mathrm{T}_{1}$ mapping using inversion recovery Look-Locker echo planar imaging at steady state: Optimization for accuracy and reliability Magn. Reson. Med. 61 899906

Treier R, Steingoetter A, Goetze O, Fox M, Fried M, Schwizer W and Boesiger P 2008 Fast and optimized $\mathrm{T}_{1}$ mapping technique for the noninvasive quantification of gastric secretion J. Magn. Reson. Imaging 28 96-102

Ungersma S E, Matter N I, Hardy J W, Venook R D, Macovski A, Conolly S M and Scott G C 2006 Magnetic resonance imaging with T1 dispersion contrast Magn. Reson. Med. 55, 1362-1371

Young I R, Clarke G J, Bailes D R, Pennock J M, Doyle F H and Bydder G M 1981 Enhancement of relaxation rate with paramagnetic contrast agents in NMR imaging J. Comput. Tomogr. 5 543-547

Zuidam N J, de Vrueh R and Crommelin D J A 2003 Characterization of liposomes, in Liposomes - $A$ Practical Approach, $2^{\text {nd }}$ edition, ed V P Torchilin and V Weissig (Oxford: Oxford University Press) p 31 